\title{
Microsatellite instability and the clinicopathological features of sporadic colorectal cancer
}

\author{
R Ward, A Meagher, I Tomlinson, T O’Connor, M Norrie, R Wu, N Hawkins
}

Department of

Medical Oncology, St

Vincent's Hospital,

Victoria St,

Darlinghurst, NSW,

2010, Australia

$\mathrm{R}$ Ward

M Norrie

$\mathrm{R}$ Wu

Department of

Colorectal Surgery, St

Vincent's Hospital,

Victoria St,

Darlinghurst, NSW,

2010, Australia

A Meagher

T O'Connor

School of Medicine, University of NSW,

Sydney, 2052, Australia

$\mathrm{R}$ Ward

M Norrie

School of Pathology, University of NSW,

Sydney, 2052, Australia

$\mathrm{N}$ Hawkins

Molecular and

Population Genetics,

Imperial Cancer

Research Fund,

Lincoln's Inn Fields,

London, WC2A 3PX,

UK

R Ward

I Tomlinson

N Hawkins

Correspondence to:

Dr R Ward, Department of

Medical Oncology, St

Vincent's Hospital, Victoria

St, Darlinghurst, 2010

Australia.

r.ward@garvan.unsw.edu.au

Accepted for publication

5 December 2000

\begin{abstract}
Background and aims-In this study, we prospectively examined the clinical significance of the microsatellite instability (MSI) phenotype in sporadic colorectal cancer, and investigated methods for effective identification of these tumours in routine pathology practice.
\end{abstract}

Methods-DNA was extracted from 310 tumours collected from 302 consecutive individuals undergoing curative surgery for sporadic colorectal cancer. Microsatellite status was determined by polymerase chain reaction amplification using standard markers, while immunostaining was used to examine expression of MLH1, MSH2, and p53.

Results-Eleven per cent of tumours showed high level instability (MSI-H), $6.8 \%$ had low level instability (MSI-L), and the remainder were stable. MSI-H tumours were significantly more likely to be of high histopathological grade, have a mucinous phenotype, and to harbour increased numbers of intraepithelial lymphocytes. They were also more likely to be right sided, occur in women, and be associated with improved overall survival. In total, $25(8 \%)$ tumours showed loss of staining for MLH1 and a further three tumours showed absence of staining for MSH2. The positive and negative predictive value of immunohistochemistry in the detection of MSI-H tumours was greater than $95 \%$.

Conclusions-We conclude that the MSI-H phenotype constitutes a pathologically and clinically distinct subtype of sporadic colorectal cancer. Immunohistochemical staining for MLH1 and MSH2 represents an inexpensive and accurate means of identifying such tumours. (Gut 2001;48:821-829)

Keywords: colorectal carcinoma; microsatellite instability; survival; MLH1; MSH2; immunohistochemistry

The phenomenon of DNA microsatellite instability (MSI) results from failure of repair of DNA mismatches immediately after DNA replication, ${ }^{1}$ and is characterised experimentally by contraction or expansion of mono-, di-, tri-, or tetranucleotide repeat sequences within DNA. ${ }^{2}$ Studies of the rare syndrome of hereditary non-polyposis colorectal cancer (HNPCC) led to recognition of MSI as the phenotypic expression of a germline mutation in one or more of the genes associated with
DNA mismatch repair. ${ }^{3-5}$ At least five of these genes have now been identified including MSH2, MLH1, hPMS1, hPMS2, and hMSH6/GTBP. ${ }^{6-9}$ However, mutations in either MLH1 or MSH2 account for the majority of known germline mutations in HNPCC families.

Colorectal carcinomas arising in the setting of HNPCC, as well as showing high level MSI (MSI-H), have a number of distinctive clinicopathological features that separate them from the sporadic forms of the disease. Importantly, individuals with HNPCC associated tumours appear to have a significantly better outcome than those with sporadic disease of similar stage and grade. ${ }^{10-12}$ They are also at greater risk of metachronous tumour development.

However, MSI is not confined to the setting of hereditary disease and may be seen in up to $15 \%$ of colorectal carcinomas in those with no family history of disease. ${ }^{13-15}$ In the case of these so-called sporadic colorectal tumours, mutation of MMR genes is relatively infrequent whereas bialleleic hypermethylation of the promoter of MLH1 appears to be the most important mechanism for inactivation of mismatch repair genes. ${ }^{16-18}$

While it is well established that a proportion of sporadic colorectal cancers demonstrate an MSI-H phenotype, the clinical significance of such a finding has not yet been determined. Some studies have shown that individuals with MSI-H tumours have improved survival relative to those with MSS tumours of similar stage. ${ }^{4-23}$ Others have not demonstrated such a survival advantage ${ }^{24-27}$ and one recent study suggested that the prognostic significance varies with site in the colon or rectum. ${ }^{28}$ Recently, a number of small retrospective studies have also suggested that individuals with MSI-H sporadic tumours are at greater risk of metachronous cancers. ${ }^{22} 232930$ Finally, there are indications that MSI-H tumours, either hereditary or sporadic, may be resistant to the actions of certain commonly used chemotherapeutic agents, ${ }^{31-33}$ raising the important possibility that neoadjuvant or adjuvant chemotherapy may have different outcomes in this group of patients.

Much of the current confusion in the literature regarding the clinical significance of these tumours has arisen because studies to date have been retrospective, and in general have been limited to relatively small populations.

Abbreviations used in this paper: MSI, microsatellite instability; MSI-H, high level MSI; MSI-L, low level MSI; MSS, microsatellite stable; HNPCC, hereditary non-polyposis colorectal cancer; PCR, polymerase chain reaction; $\mathrm{HR}$, hazard ratio. 
Furthermore, they have often been conducted in cancer dense families, or in selected subgroups of sporadic disease such as individuals with an early onset disease or with multiple primary tumours. ${ }^{21-23}$

Adding to this confusion, it has recently been proposed that sporadic colorectal carcinoma can be categorised into three distinct groups, with either high (MSI-H), low (MSI-L), or absent (microsatellite stable, MSS) levels of microsatellite instability. ${ }^{15}$ In the case of MSI-L sporadic tumours, little is known of the prognostic implications or risk of metachronous tumour development. ${ }^{23}$ Nevertheless, it has been proposed that the MSI-L phenotype reflects a distinctive pathway of tumour development that may be reflected in altered clinical behaviour. ${ }^{34}$

In this study, we prospectively examined the clinical significance of these genetic subtypes of sporadic colorectal cancer and evaluated the optimal methods for identification of these tumours.

\section{Materials and methods}

PATIENTS AND SPECIMENS

After obtaining informed consent, 302 consecutive individuals undergoing surgical resection of adenocarcinoma of the colon or rectum at St Vincent's Hospital, Sydney, were enrolled in this prospective study from 1993 to 1998. Individuals were excluded where preoperative radiotherapy or chemotherapy had been administered. Patients with inflammatory bowel disease or with a known history of familial adenomatous polyposis or HNPCC were also excluded from this study, as were those individuals in whom the primary tumour was incompletely resected (R1 or R2). ${ }^{35}$

Fresh representative tissue samples $(500 \mu \mathrm{g})$ from all tumours and paired normal colonic mucosa were immediately frozen at $-70^{\circ} \mathrm{C}$. In total, 310 fresh tumour specimens were assayed from 172 males and 130 females (aged 29-95 years; mean 68.4 (12.4)). Fifty one of these tumours were TNM stage I while 113 were stage II, 97 were stage III, and 49 were stage IV.

Family histories of colorectal carcinoma and other malignancies were obtained by interviewing patients or their next of kin. Attempts were made to verify all suspected diagnoses of cancer and uncertain causes of death, either by obtaining death certificates and medical records or by contacting the treating physician. Family history was used to identify those families that met either the Amsterdam or modified Amsterdam criteria for HNPCC. ${ }^{36}$

HISTOPATHOLOGICAL ANALYSIS OF TUMOURS

For all tumours, histopathological type, stage, and size were determined independently by a histopathologist within the Department of Anatomical Pathology, St Vincent's Hospital. Tumour grade, extent of mucin production, tumour growth pattern, as well as the presence of a Crohn's-like inflammatory infiltrate, intraepithelial lymphocytes, or peritumoural lymphocytes were determined prospectively without knowledge of the mismatch repair status. Tumours in which less than $10 \%$ of cells formed glands were classified as high grade (poorly differentiated) while those containing more than $50 \%$ extracellular mucin were classified as mucinous. ${ }^{37}$ The tumour growth pattern was interpreted as either infiltrative or expansile, according to previously published criteria. ${ }^{38}$ The extent of peritumoural and Crohn's-like lymphoid reactions was classified according to the method of Jass and colleagues. ${ }^{38}$ Intraepithelial lymphocytes were identified by light microscopy on haematoxylin and eosin sections as cells with the morphology of lymphocytes, seen wholly within tumour epithelium. They were classified as conspicuous when more than 30 were present per 10 high power fields.

Tumour volume in this study was estimated from reported tumour dimensions, using the formula $\mathrm{V}=\pi((\mathrm{L}+\mathrm{T}) / 4)^{2} \times \mathrm{D}$, where $\mathrm{V}=$ volume $(\mathrm{ml}) ; \mathrm{L}=$ longitudinal dimension $(\mathrm{cm}) ; \mathrm{T}=$ circumferential dimension $(\mathrm{cm})$; and $\mathrm{D}=$ depth of tumour $(\mathrm{cm})$.

DNA EXTRACTION

For preparation of DNA from fresh tissues, frozen tissue was macerated in $500 \mu \mathrm{l}$ of ice cold lysis buffer $(10 \mathrm{mM}$ Tris $\mathrm{HCl}, 1 \mathrm{mM}$ EDTA, $100 \mathrm{mM} \mathrm{NaCl}, 1 \%$ sodium dodecyl sulphate, $500 \mu \mathrm{g} / \mathrm{ml}$ proteinase $\mathrm{K}$ ) using a sterile Eppendorf homogeniser. Following incubation overnight with shaking at $50^{\circ} \mathrm{C}$, DNA was extracted with phenol/chloroform and precipitated with ethanol.

For extraction of DNA from paraffin embedded tissues, $4 \times 5 \mu \mathrm{m}$ sections from the tissue block were incubated in $250 \mu \mathrm{l}$ of paraffin lysis buffer $\left(50 \mathrm{mM} \mathrm{KCl}, 2.5 \mathrm{mM} \mathrm{MgCl}_{2}, 0.45 \%\right.$ Triton-X, $10 \mathrm{mM}$ TRISMA base, $500 \mu \mathrm{g} / \mathrm{ml}$ proteinase $\mathrm{K}$ ) for two hours at $65^{\circ} \mathrm{C}$ and then overnight at $55^{\circ} \mathrm{C}$. The tubes were then boiled for five minutes, centrifuged at $14000 \mathrm{~g}$ for two minutes, and finally chilled prior to removal of the DNA solution from beneath the solidified paraffin. For each tumour block an adjacent section was stained with haematoxylin and eosin, and examined by light microscopy to ensure that the section contained more than $60 \%$ tumour tissue.

\section{ANALYSIS OF MICROSATELLITE INSTABILITY}

In all reactions, DNA was amplified in a $10 \mu \mathrm{l}$ volume containing $100 \mathrm{ng}$ of DNA, $200 \mu \mathrm{M}$ dNTPs, $1.5 \mathrm{mM} \mathrm{MgCl}_{2}, 0.27 \mu \mathrm{M}$ of each primer, $0.25 \mathrm{U}$ T th polymerase in a buffer of $16.6 \mathrm{mM}(\mathrm{NH} 4)_{2} \mathrm{SO} 4,0.45 \%$ Triton X-100, $0.2 \mathrm{mg} / \mathrm{ml}$ gelatin, and $67 \mathrm{mM}$ Tris $\mathrm{HCl}, \mathrm{pH}$ 8.8 (Biotech International, Ltd, Western Australia). The reactions were incubated at $95^{\circ} \mathrm{C}$ for five minutes followed by 35 cycles of $95^{\circ} \mathrm{C}$, $57^{\circ} \mathrm{C}$, and $72^{\circ} \mathrm{C}$ for one minute each. The primers used in this study were Bat25, Bat26, D5S346, D2S123 and D17S250. ${ }^{39}$ Products were run on an ABI 377 sequencer (Foster City, California, USA) and analysed using Genescan and Genotyper software.

MSI was defined as either marked alteration in repeat length (often appearing as a "ladderlike" expansion) or as a new discrete band 
above or below the expected allele. Small increases in amplitude or numbers of stutters in the tumour samples were not considered to represent instability at the marker. A single observer (RW) performed analysis of MSI and equivocal samples were re-evaluated by a second observer (IT). Results were reported without knowledge of pathological or immunohistochemical status. A tumour sample was considered to be MSI-H if two or more of the markers demonstrated instability and to be MSI-L when only one marker was unstable. ${ }^{39}$ In some cases MSI analysis was repeated using DNA extracted from paraffin embedded tissue. This was performed where the individual had synchronous cancers and the origin of fresh samples was uncertain, where polymerase chain reaction (PCR) amplification of DNA from fresh tissue was not satisfactory, or where there were indeterminate results from one or more markers. Overall, analysis of paraffin extracted DNA was necessary to obtain reliable data in 29 tumours.

IMMUNOHISTOCHEMICAL STAINING FOR MSH2 AND MLH1

Following dewaxing and rehydration of $4 \mu \mathrm{m}$ tissue sections on silane coated slides, endogenous peroxidase was blocked using 3\% hydrogen peroxide in methanol for 20 minutes. Antigen retrieval was performed by pressure cooking for five minutes in $0.1 \mathrm{M}$ citrate buffer (pH 6.0). Slides were then blocked with $20 \%$ normal horse serum diluted in $2 \%$ bovine serum albumin/Tris buffered saline prior to overnight incubation with monoclonal antibodies against $\mathrm{MSH} 2(1: 400)$ and $\mathrm{MLH} 1$ (1:200) (Pharmingen, San Diego, California, USA). Bound antibody was detected with biotinylated horse antimouse immunoglobulin (1:100; Dako, Dakopatts, Denmark) and horseradish peroxidase labelled streptavidin (SA-HRP, 1:200; Dako). Staining was considered assessable where nuclear staining was seen in either stromal or germinal follicle lymphocytes, or in normal epithelial cells in the base of crypts. In assessable slides, complete absence of staining of tumour cells for one of the mismatch repair proteins in the presence of staining for the other protein was considered indicative of a mismatch repair defect.

ANALYSIS OF SOMATIC CHANGES IN p53 AND K-ras Mutations at the first and second bases of codon 12 of the K-ras gene were detected using restriction endonuclease mediated selective PCR, as previously described..$^{40}$ For identification of accumulation of p53 within tumour cells, paraffin sections of tumour tissue were subjected to immunohistochemical analysis of p53, as previously described, ${ }^{41}$ using the mouse antihuman p53 antibody DO7 (Dako). Tumour was considered to show accumulation of p 53 protein when more than $20 \%$ of tumour cells showed nuclear staining of moderate to high intensity, in the absence of staining in the stromal cells and normal epithelium.
STATISTICAL ANALYSIS

Survival was measured from the date of resection of colorectal cancer until death or until the censor date of 1 March 2000. Time to recurrence was the period from resection to medical documentation of tumour recurrence. Survival and time to recurrence were censored at time of death for patients dying of causes other than colorectal cancer. Survival curves were prepared according to the method of Kaplan and Meier, and statistical significance between curves was tested using the log rank test. Univariate survival analysis was performed by Cox proportional hazards model, and the hazard ratio and its $95 \%$ confidence interval (CI) were assessed for each factor. A multivariate analysis by stepwise backward conditional selection was used to determine which factors were independent prognostic factors for survival. For the 210 individuals alive at the time of the last follow up, the median follow up period was 32 months (range 1-74).

Categorical variables were compared using the $\chi^{2}$ test or Fisher's exact test as appropriate. For comparison of MSI status, the Student $t$ test was used for analysis of patient age, Wilcoxson rank sum test for analysis of tumour volume, and Spearman's test for tumour stage. A probability value of less than 0.05 was considered significant. All data were analysed using the SPSS statistical software v9.0 (SPSS Inc., Chicago, Illinois, USA).

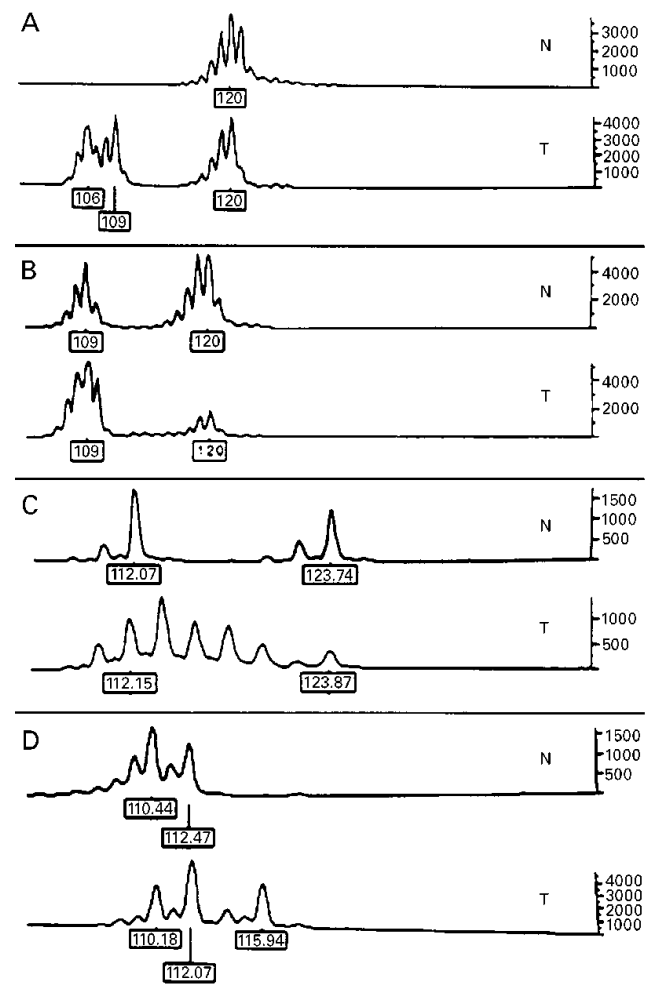

Figure 1 Microsatellite analysis of paired normal $(N)$ and tumour (T) DNA using Bat $26(A, B)$, and D5S346 $(C, D)$. The tumours in $(A),(C)$, and $(D)$ are unstable. The tumour in (B) (stable) has a germline polymorphism that is only identifiable as such by the presence of the same pattern in normal tissue. (C) (laddering) and (D) (new allele) are representative of the patterns of changes observed at the dinucleotide markers. Size in base pairs is indicated, and the scale on the right shows signal amplitude. 
Table 1 Comparison of differences in a range of clinicopathological characteristics between MSI-H and MSS colorectal carcinomas. MSS tumours include the MSI-L group. Except where indicated ('Spearman's), significance was determined by Fisher's exact test (two tailed)

\begin{tabular}{|c|c|c|c|c|c|}
\hline & \multicolumn{2}{|c|}{ MSI } & \multirow[b]{2}{*}{ p Value } & \multirow[b]{2}{*}{$P P V$} & \multirow[b]{2}{*}{$N P V$} \\
\hline & + & - & & & \\
\hline \multicolumn{6}{|c|}{ Right sided tumour } \\
\hline+ & 26 & 89 & $<0.001$ & 0.23 & 0.96 \\
\hline - & 7 & 188 & & & \\
\hline \multicolumn{6}{|c|}{ Intraepithelial lymphocytes } \\
\hline+ & 22 & 41 & $<0.001$ & 0.35 & 0.96 \\
\hline \multirow{2}{*}{\multicolumn{6}{|c|}{ Poor differentiation }} \\
\hline & & & & & \\
\hline+ & 11 & 31 & 0.002 & 0.26 & 0.92 \\
\hline - & 22 & 246 & & & \\
\hline \multicolumn{6}{|c|}{ Crohn's-like reaction } \\
\hline+ & 11 & 32 & 0.002 & 0.26 & 0.91 \\
\hline - & 22 & 235 & & & \\
\hline \multicolumn{6}{|c|}{ Mucinous tumour } \\
\hline+ & 10 & 40 & 0.022 & 0.20 & 0.91 \\
\hline - & 22 & 235 & & & \\
\hline \multicolumn{6}{|c|}{ Peritumoural lymphocytes } \\
\hline+ & 16 & 52 & $<0.001$ & 0.24 & 0.93 \\
\hline- & 17 & 225 & & & \\
\hline \multicolumn{6}{|c|}{ Circumscribed margin } \\
\hline+ & 29 & 198 & 0.059 & 0.13 & 0.95 \\
\hline - & 4 & 79 & & & \\
\hline \multicolumn{6}{|c|}{ Tumour stage } \\
\hline I & 5 & 46 & $0.27^{\mathrm{a}}$ & & \\
\hline II & 17 & 96 & & & \\
\hline III & 7 & 90 & & & \\
\hline IV & 4 & 45 & & & \\
\hline \multicolumn{6}{|l|}{ Sex } \\
\hline $\mathrm{F}$ & 21 & 107 & 0.004 & & \\
\hline M & 10 & 162 & & & \\
\hline \multicolumn{6}{|c|}{ Amsterdam criteria } \\
\hline No & 30 & 266 & 0.35 & 0.25 & 0.90 \\
\hline Yes & 1 & 3 & & & \\
\hline
\end{tabular}

MSI, microsatellite instability; MSI-H, high level of microsatellite instability; MSI-L, low level of microsatellite instability; MSS, microsatellite stable; PPV, positive predictive value, NPV, negative predictive value.
D5S346 in $52 \%$, D2S 123 in $30 \%$, and D $17 \mathrm{~S} 250$ in $20 \%$ of cases.

PATHOLOGICAL, GENETIC, AND CLINICAL CORRELATES OF MICROSATELLITE INSTABILITY Statistical analysis showed that there were no significant differences between MSI-L and MSS tumours in any of the clinicopathological characteristics examined in this study. In contrast, MSI-L tumours differed significantly from MSI-H tumours in terms of right sidedness $(p<0.001)$ and presence of intraepithelial lymphocytes $(p=0.01)$. On this basis, MSI-L tumours were included in the MSS category for all subsequent analysis of microsatellite status and its relationship to clinicopathological and genetic variables.

MSI-H tumours were significantly more likely to be of high histopathological grade, have a mucinous phenotype, and to harbour increased numbers of both intraepithelial and peritumoural lymphocytes. They were also more likely to be right sided and to occur in women (table 1). Although the MSI-H phenotype appeared to be over represented in stage II tumours, this association did not reach statistical significance. There was no association between MSI-H status and patient age or family history, as determined by the Amsterdam criteria (table 1) or modified Amsterdam criteria (data not shown).

Positive and negative predictive values were calculated for each pathological parameter (table 1) as well as for all possible simple combinations of four of these values (sidedness, intraepithelial lymphocytes, grade, and margin type). On this basis, it was apparent that no individual pathological parameter had a clinically useful positive predictive value. In simple combination, the presence of both right sidedness and intraepithelial lymphocytes was the most accurate predictor of MSI-H phenotype, with a positive predictive value of $57 \%$ and a negative predictive value of $95 \%$.

In general, MSI-H tumours had a greater tumour volume, although this was not statistically significant (MSS median $13 \mathrm{ml}$, range 0-567; MSI-H median $25 \mathrm{ml}$, range 1-187; $\mathrm{p}=0.06$ ). When considered by stage, this effect was not present in stage I tumours but was present in stage II tumours (MSS median 15 $\mathrm{ml}$, range 1-448; MSI-H median $24 \mathrm{ml}$, range $6-110 ; p=0.20)$ and most marked in stage III tumours (MSS median $12 \mathrm{ml}$, range 1-232; MSI-H median $31 \mathrm{ml}$, range $1-70 ; \mathrm{p}=0.12$ ).

Sixteen individuals in the study had either synchronous (10 individuals) or metachronous (seven individuals) colorectal carcinomas (one individual had both synchronous and metachronous tumours). One individual had two synchronous MSI-H tumours while another had simultaneous MSI-H and MSS carcinomas. A further individual with an MSI-H tumour had two metachronous colorectal carcinomas. The remaining 13 individuals were MSS and there was no significant increase in the frequency of metachronous or synchronous tumours in those with MSI-H tumours $(\mathrm{p}=0.39)$. all MSI-L tumours were stable at Bat 26 and Bat25, and the predominant change in the dinucleotide markers was the appearance of a new allele $(50-60 \%$ of cases). In MSI-L tumours, the unstable marker was found to be 

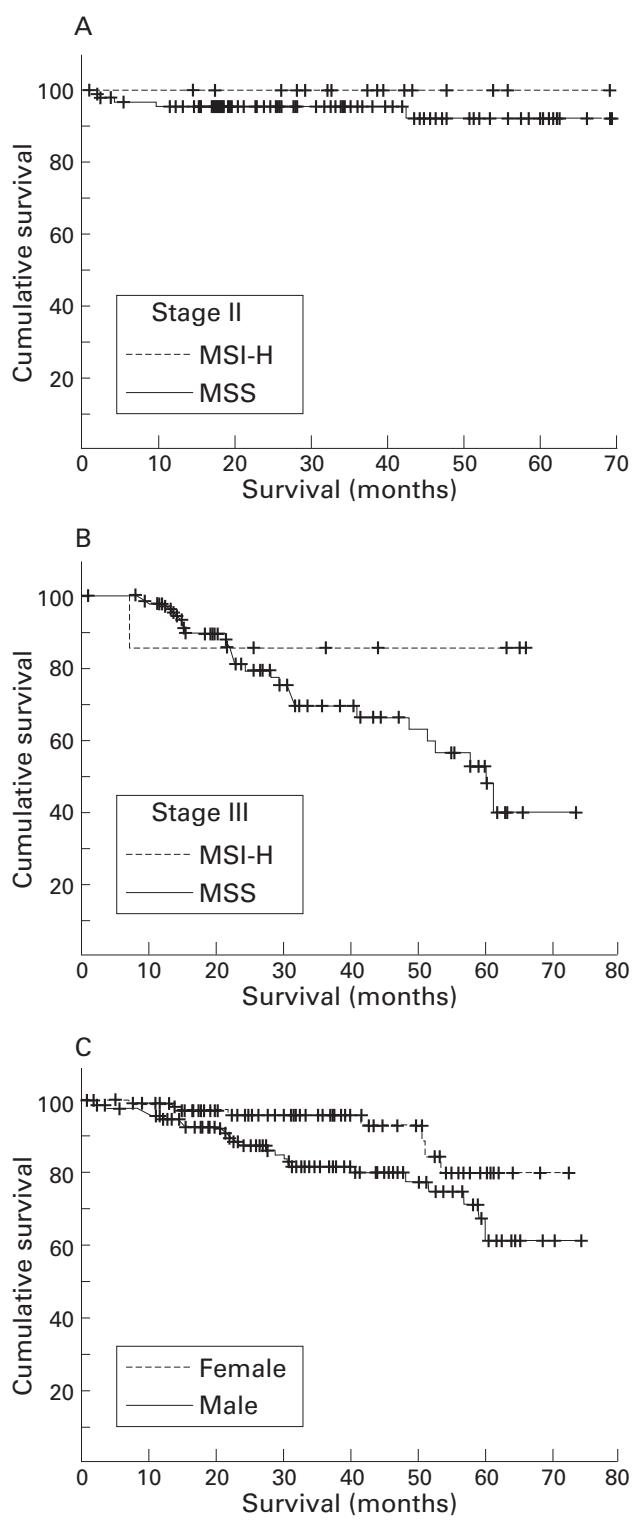

Figure 2 Kaplan-Meier survival curves for individuals with colorectal carcinoma. $(A, B)$ Survival according to microsatellite instability status in stage II and stage III tumours, respectively. (C) Overall survival as a function of sex. MSI-H, high level of microsatellite instability; MSS, microsatellite stable.

K-ras mutations were detected in 82 of 300 tumours analysed $(27 \%)$ while immunohistochemical evidence of p53 accumulation was observed in 157 of 309 tumours (50.8\%). While the incidence of K-ras mutations was less frequent in tumours with MSI (16\%) than in either MSI-L (29\%) or stable tumours (29\%), this difference was not significant $(\mathrm{p}=0.20)$. On the other hand, accumulation of $\mathrm{p} 53$ was much less common in MSI-H cancers (18\%) than in non-MSI- cancers $(55 \%)(\mathrm{p}<0.001)$.

\section{DISEASE OUTCOMES AND MICROSATELLITE}

STATUS

Analysis of overall survival and time to recurrence was performed on all individuals who had undergone potentially curative tumour resection (stages I-III, R0).

Cox's univariate analysis showed that female sex (hazard ratio (HR) 0.4 (CI 0.17-0.87); $\mathrm{p}=0.02$ ), lower tumour stage (HR 4.62 (CI 2.3-9.5); $\mathrm{p}<0.0001)$, peritumoural lymphocytes (HR 0.24 (CI 0.07-0.77); p=0.02), circumscribed tumour margins (HR 3.46 (CI $1.75-6.8) ; \mathrm{p}<0.003)$, and increased intraepithelial lymphocytes (HR 0.29 (CI 0.09-0.95); $\mathrm{p}=0.04$ ) were each associated with improvement in overall survival. No survival differences were accorded by any other pathological factor, MSI status, or by the presence of p53 accumulation or K-ras mutation within the tumour. In the multivariate analysis, tumour stage (HR 4.34 (CI 2.1-8.77); p<0.001) and female sex (HR 0.41 (CI 0.18-0.91); $\mathrm{p}=0.03$ ) proved to be the only independent predictors of improved survival.

Survival curves for patients with stage II and III cancers according to microsatellite status, as well as overall survival by sex, are shown in fig 2. Although the differences between microsatellite groups did not reach statistical significance it is apparent that individuals with MSI-H tumours showed a trend towards both a prolonged recurrence free interval and improved overall survival. It is notable that of the 17 patients with stage II cancer, none had developed recurrent disease or died from cancer as of the censor date.

In relation to survival, it is also important to note that there were an increased number of stage II tumours in the MSI-H group, and a concomitant decrease in relative terms in stages III and IV (table 1). However, these differences in stage distribution did not reach statistical significance and were not reflected in improved overall survival.

\section{DETECTION OF MSI USING IMMUNOHISTO-} CHEMISTRY

The typical appearance of tumours stained with antibodies against MLH1 and MSH2 is shown in fig 3. Immunohistochemistry could not be interpreted in two tumours, both from the MSS group, because of failure of staining of normal cells. Overall, 25 (8\%) tumours showed loss of staining for MLH1 and a further three tumours $(1 \%)$ showed absence of staining for MSH2. No tumour showed loss of staining with both antibodies.

There was a highly significant correlation between MSI-H status and loss of expression of MSH2 or MLH1 $(\mathrm{p}<0.001)$ (table 2). In fact, immunohistochemical analysis with MLH1 and MSH2 offered a specificity of $99.6 \%$ for detection of MSI as only one of 275 MSS tumours was reported to show loss of expression of a mismatch repair enzyme (IHC false positive). The sensitivity of the immunohistochemical analysis was $81 \%$ as it was unable to detect six of $33 \mathrm{MSI}-\mathrm{H}$ tumours (IHC false negatives). The positive predictive value of immunohistochemistry in the detection of MSI-H tumours was calculated to be $96 \%$ and the negative predictive value was $98 \%$.

The one IHC false positive tumour was MSI-L by genetic testing. However, this was determined using fresh tissue and it is possible that there may have been insufficient tumour in 


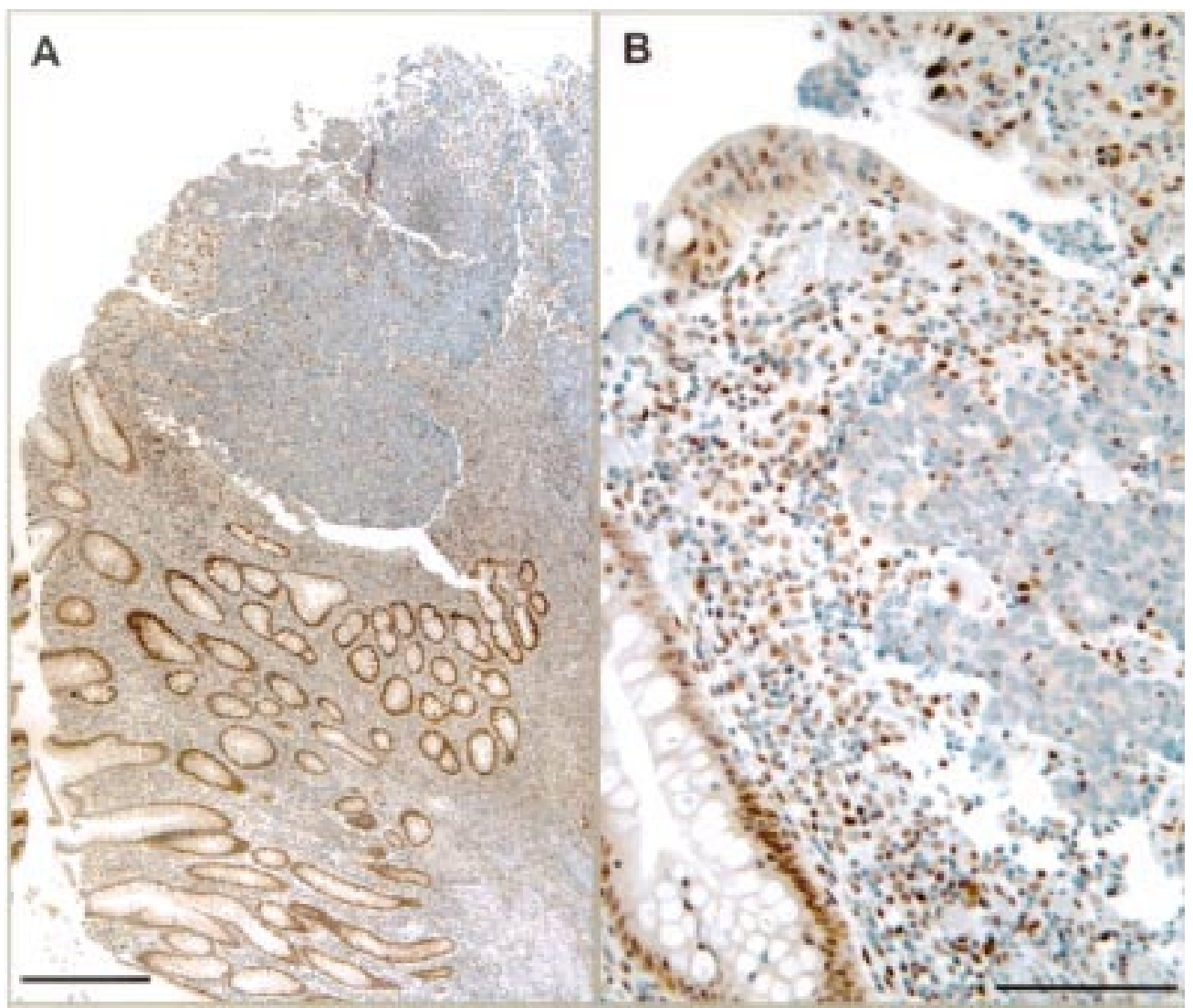

Figure 3 Typical appearance of tumours showing loss of mismatch repair enzymes, as demonstrated by immunohistochemistry for MLH1. (A) Poorly differentiated carcinoma cells (upper) show no nuclear staining for MLHI while normal crypt epithelium (lower left) and stromal lymphocytes show nuclear positivity (brown stain); bar=500 $\mu \mathrm{m}$. (B) Details of negative tumour cell staining in the presence of dark staining positive lymphocytes, both within tumour epithelium and in surrounding stroma. Normal epithelium is seen in the lower left; bar $=100 \mu \mathrm{m}$; immunoperoxidase with haematoxylin counterstain.

Table 2 Comparison of microsatellite analysis with MSH2 and /MLH1 immunohistochemical findings. Mismatch repair defective tumours are those carcinomas which showed loss of either MLH1 or MSH2 expression by immunohistochemistry

\begin{tabular}{lrrr}
\hline & \multicolumn{2}{c}{ MSI } & \\
\cline { 2 - 3 } & \multicolumn{2}{c}{ MSS } & \multicolumn{2}{c}{ MSI-H } & Total \\
\hline MLH1 and MSH2 present & 274 & 6 & 280 \\
MLH1 loss & 1 & 24 & 28 \\
MSH2 loss & 0 & 3 & 3 \\
Total & 275 & 33 & 308 \\
\hline
\end{tabular}

MSI, microsatellite instability; MSI-H, high level of microsatellite instability; MSS, microsatellite stable.

the sample to ensure accurate analysis of all markers.

The clinicopathological features of the six IHC false negative tumours are shown in table 3 . While most cases have the typical morphology of MSI-H tumours it is interesting that two cases in the transverse colon did not show increased levels of intraepithelial lymphocytes or mucinous phenotype, were moderately differentiated, and had mutations of p53. In terms of microsatellite analysis, one of these two cases (FN3) was unstable only at the BAT25 and BAT26 while the other case (FN4) was unstable only at D5S346 and D2S123. The other four cases showed instability at three or more microsatellite loci, including BAT markers. Review of immunohistochemical data confirmed the fact that they all expressed both MSH2 and MLH1 protein.

Based on clinical data, in combination with immunohistochemical and genetic analysis of tumours, it was possible that up to six individuals in this study with apparently sporadic MSI-H tumours may actually harbour germline mutations of a mismatch repair gene.

Table 3 Characteristics of individuals and tumours with MSI-H tumours but with no mismatch repair defect, as determined by immunostaining (false negative IHC)

\begin{tabular}{lllllllllll}
\hline ID & Age & Sex & AC & Stage & Site & IEL & Mucin & Grade & p53 & K-ras \\
\hline FN1 & 62 & F & No & I & AsC & + & No & Mod & Wild & Mut \\
FN2 & 62 & M & No & II & AsC & + & Yes & Poor & Wild & Wild \\
FN3 & 67 & F & No & II & TC & - & No & Mod & Mut & Wild \\
FN4 & 77 & F & No & II & TC & - & No & Mod & Mut & Wild \\
FN5 & 83 & F & No & IV & Caecum & + & Yes & Poor & Wild & Wild \\
FN6 & 84 & F & No & IV & SC & - & No & Poor & Wild & Wild \\
\hline
\end{tabular}

AC, Amsterdam criteria, IEL, intraepithelial lymphocytes, Mut, mutant, TC, transverse colon, AsC, ascending colon, SC, sigmoid colon; MSI-H, high level of microsatellite instability.

*This patient had three first degree relatives with either breast or gastric carcinoma. 
These included three individuals with tumours that were negative for MSH2 by immunohistochemistry (aged 46, 72, and 80 years) as somatic mutations of this gene are reported to be infrequent in sporadic MSI-H cancers. ${ }^{15}{ }^{17}$ Interestingly, none of these three individuals fulfilled the Amsterdam criteria. Alternatively, reduced expression of $\mathrm{MSH} 2$ in these tumours may have been secondary to another genetic defect. ${ }^{20}$ A further two individuals with MLH1 negative tumours were considered likely to represent individuals with germline disease as one was aged 32 years at the time of surgery while the other (aged 67) fulfilled the Amsterdam criteria. Finally, a sixth individual had a personal history of three colorectal carcinomas from age 38 as well as an MLH1 negative tumour. She was shown by subsequent genetic testing to have a germline mutation at exon 9 of MLH1 even though she did not fulfill the Amsterdam criteria.

When individuals likely to have a germline defect were excluded from the analysis, the remaining 21 individuals with MSI-H tumours that lacked MLH1 expression differed from other individuals in the study in that they were older (mean age 76 (9) years; $p=0.004$ ) and were more likely to be female ( $66 \%$ female; $\mathrm{p}=0.04)$.

\section{Discussion}

It is now well accepted that a significant proportion of sporadic colorectal carcinomas arise through a pathway that is characterised by defects in DNA repair enzymes and that is defined phenotypically by MSI. The extent and significance of this phenomenon in sporadic disease remain less well delineated.

Accrual of accurate data on the frequency and significance of MSI in sporadic colorectal cancer must be predicated on the unbiased selection of cases. It is now established that the rate of MSI-H tumours is relatively high in certain selected populations, including younger individuals $\left(17-58 \%{ }^{42-45}\right.$ ) and those with mucinous tumours $\left(36 \%{ }^{24}\right)$. In the case of the younger population, this is almost certainly related to a significantly higher frequency of germline defects. ${ }^{45}$ In this study, we have examined the frequency and significance of MSI in a prospective fashion from a population of individuals with apparently sporadic colorectal carcinoma. It is inevitable that some individuals enrolled in this study with apparent sporadic disease were in fact harbouring germline mutations and we estimate that up to six individuals in this study may fall into this category. Nevertheless, for the purposes of this study, all individuals were considered to represent sporadic disease, in the sense that they presented for surgery and pathological assessment of their tumour without any expectation by the relevant medical staff that their condition represented a hereditary disease. In practical terms, we believe that this is the "sporadic" population that confronts the surgeon, pathologist, or oncologist outside centres with an interest in hereditary disease, and the population from which data on the value and significance of MSI is most urgently needed.
PATHOLOGICAL AND GENETIC FEATURES OF MSI IN SPORADIC COLORECTAL CARCINOMA

Many of the observations made in this study regarding the frequency, phenotype, and genotype of sporadic MSI-H tumours are in concordance with previously published studies. ${ }^{13}{ }^{23}$ We found that MSI-H tumours were more frequent in the right colon, that they often had a mucinous and poorly differentiated phenotype, and that intraepithelial and peritumoural lymphocytic infiltration were often prominent. In common with others, we found that MSI-H tumours associated with apparent somatic loss of MLH1 were more common in the elderly and in women, and that there was a strong correlation between MSI-H and absence of p53 mutations, as determined by immunostaining. ${ }^{13} 46$

However, MSI-H tumours were seen in only $10.6 \%$ of this unselected population, a rate that is lower than any previously reported study. ${ }^{22}{ }^{23}$ For the reasons indicated above, we consider that this number may represent a more accurate estimate than has previously been reported in the literature on sporadic MSI-H colorectal carcinoma. Similar to many other reports, we found that mononucleotide repeat markers provided the most sensitive and specific single marker of instability. ${ }^{15} 47$

As a result of this work, we are also able to comment on the significance of the MSI-L phenotype in sporadic colorectal carcinoma. It has previously been proposed that the MSI-L phenotype reflects a distinctive pathway of tumour development, ${ }^{34}$ involving sequences other than mononucleotide repeats. ${ }^{15}$ While data from this study support the association of the MSI-H phenotype with a range of characteristic pathological features, the same cannot be said of the MSI-L group of cancers. In fact, we were unable to find any clinicopathological features which distinguished this group of cancers from the MSS group. On the other hand, MSI-L tumours showed clear pathological differences from MSI-H tumours in a number of areas.

CLINICAL SIGNIFICANCE OF MSI IN THE SPORADIC SETTING

Detection of MSI in the setting of hereditary disease appears to identify individuals at increased risk of second tumours, as well as those with an improved survival compared with MSS tumours. To date, information on these clinical parameters has been less clear in the setting of sporadic disease.

Several small and retrospective studies have suggested that individuals with MSI-H sporadic tumours are at greater risk of metachronous cancers. ${ }^{22} 2930$ More recently, Gryfe and colleagues found a higher incidence of both metachronous and synchronous tumours in younger patients with MSI-H cancers, a study which is likely to have included more patients with germline mismatch repair defects. ${ }^{45}$ However, we were unable to show an increase in the risk of such tumours in our study group, which consisted largely of older individuals. 
The relationship between MSI status and disease outcomes in sporadic colorectal carcinoma remains uncertain. In this study, patients with MSI-H cancers showed a trend towards improved clinical outcome, and it is possible that as the period of follow up matures, these differences will reach statistical significance. Results from other studies on unselected populations, in combination with our own, suggest that MSI-H tumours have a survival advantage. ${ }^{48}$

Interestingly, the most compelling evidence to date for increased survival in sporadic MSI-H tumours has come from studies of young individuals. ${ }^{45}$ It is possible that the discrepancies in reported outcomes seen in recent studies may reflect a biological distinction between old and young individuals with microsatellite unstable tumours. In this regard, it has been proposed that MSI may influence survival through induction of an antitumour response, as evidenced by the marked lymphocytic infiltrate in the tumour. It is tempting to speculate that the apparent improved survival seen in younger people with MSI-H tumours may be related to a more intense host immune response in these individuals.

A further consideration in assessing survival is the tendency for MSI-H tumours to fail to progress from stage II to III. This is manifest in this study by the increased percentage of stage II tumours, decreased percentage of stage III tumours, and also by the observation that MSI tumours tend to be larger than stable tumours in all stages except for stage I. The biological basis of this phenomenon is not clear from this study but may be related to the low frequency of p53 mutations in MSI tumours, perhaps resulting in inhibition of tumour progression. Importantly, if delayed progression from local (stage II) to metastatic (stage III) disease is a feature of MSI tumours, it may result in an overall survival advantage, even in the absence of stage specific improvements in survival.

ALTERNATE METHODS FOR DETECTION OF MSI TUMOURS

While PCR amplification of microsatellite repeats remains the gold standard for recognition of the MSI phenotype, this approach is clearly not feasible in routine pathology laboratories. Nevertheless, the effectiveness of morphological and immunohistochemical analysis in the prospective identification of sporadic tumours remains uncertain. An important aim of this study was to identify the most appropriate methods for detection of MSI-H tumours in the setting of routine pathology practice.

It is recognised that sporadic MSI-H colorectal cancers share a number of the pathologically important features of hereditary disease, and it has been suggested that careful observation of tumour morphology can assist in the identification of these unstable tumours. ${ }^{49}$ It is clear from this study that while some morphological parameters are useful in identifying those tumours that are unlikely to be MSI-H, the same is not true for the identification of tumours likely to be MSI positive. Based on data from this study, a simple scoring that considered the presence of both right sidedness and intraepithelial lymphocytes had a positive predictive value of $57 \%$ and a negative predictive value of $95 \%$ (table 1 ). However, such a strategy would miss 13 of the 33 MSI high tumours identified in this study.

In contradistinction to morphology, we found that immunohistochemical detection of loss of expression of mismatch repair enzymes provided a highly specific approach to the identification of microsatellite unstable tumours (positive and negative predictive values $96 \%$ and $97.8 \%$, respectively). Unlike PCR analysis for MSI, it also provides a rapid and technically simple approach to this problem.

Recently, Cawkwell and colleagues provided evidence for the clinical utility of this approach in a retrospective analysis of a highly selected phenotypic subgroup of tumours. On the basis of this work, they concluded that all MSI-H cancers could be detected by immunostaining. ${ }^{22}$ However, the current study clearly identified six tumours that were MSI-H yet had apparently normal expression of MLH1 and MSH2 by immunostaining. A similar false negative rate for immunohistochemistry has previously been reported $\mathrm{d}^{50}$ and is probably multifactorial in origin.

One possible explanation is that missense mutation of a DNA repair enzyme gene leads to expression of an immunologically reactive yet poorly functional protein. A second possibility is that these false negative tumours harbour mutations in other mismatch repair genes, or in as yet unidentified genes that affect MMR function. For example, hMSH6 mutations have been reported in HNPCC and sporadic colorectal carcinomas with $\mathrm{MSI}-\mathrm{H} .^{52}$ Despite these findings, the role of hMSH6 remains to be determined as these MSI-H tumours also had mutations in other mismatch repair genes, and a recent study using colorectal cancer cell lines identified two cell lines with mutations in hMSH6 but with no MSI. ${ }^{53}$ Regardless of the genetic aetiology, we feel that such MSI-H tumours with normal immunostaining represent a small but important group of tumours which warrant further study.

In this study, we have examined MSI in a large group of colorectal cancer patients with apparently sporadic disease. On the basis of this work, it appears feasible to incorporate immunostaining as part of the standard assessment of colorectal tumours. This knowledge is of considerable relevance to the future management strategies for all individuals with microsatellite unstable cancers.

The authors wish to acknowledge Catherine Sheehan, David Wanigesekera from St Vincent's Hospital, Andy Davies and the staff of the Equipment Park, Imperial Cancer Research Fund for their technical assistance, and Professor Rodney Scott for helpful discussions. Grant support was provided by St Vincent's Clinic Foundation. RLW was supported by the Macquarie Bank-Royal Australian College of Physicians Fellowship and the Bank-Royal Australian College of Physicians Fellowship and the Astra Grant-in-Aid Gastroenterology Society of Australia. MN is supported by a postgraduate scholarship from the National
Health and Medical Research Council.

1 Modrich P. Mechanisms and biological effects of mismatch repair. Annu Rev Genet 1991;25:229-53.

2 Kolodner RD, Marsischky GT. Eukaryotic DNA mismatch repair. Curr Opin Genet Dev 1999;9:89-96. 
3 Ionov Y, Peinado MA, Malkhosyan S, et al. Ubiquitous somatic mutations in simple repeated sequences reveal a new mechan

4 Thibodeau SN, Bren G, Schaid D. Microsatellite instability in cancer of the proximal colon. Science 1993;260:816-19

5 Peltomaki P, Aaltonen LA, Sistonen P, et al. Genetic mapping of a locus predisposing to human colorectal cancer. Science 1993;260:810-12.

6 Fishel R, Lescoe MK, Rao MR, et al. The human mutator gene homolog MSH2 and its association with hereditary nonpolyposis colon cancer. Cell 1993;75:1027-38

7 Nicolaides NC, Papadopoulos N, Liu B, et al. Mutations of two PMS homologues in hereditary nonpolyposis colon cancer. Nature 1994;371:75-80.

8 Papadopoulos N, Nicolaides NC, Wei YF, et al. Mutation of a mutL homolog in hereditary colon cancer. Science 1994; 263:1625-9.

9 Papadopoulos N, Nicolaides NC, Liu B, et al. Mutations of GTBP in genetically unstable cells. Science 1995;268:191517.

10 Jarvinen HJ, Mecklin JP, Sistonen P. Screening reduces colorectal cancer rate in families with hereditary nonpolyposis colorectal cancer. Gastroenterology 1995;108:140511.

11 Sankila R, Aaltonen LA, Jarvinen HJ, et al. Better survival rates in patients with MLH1-associated hereditary colorectal cancer. Gastroenterology 1996;110:682-7.

12 Aarnio M, Mustonen H, Mecklin JP, et al. Prognosis of colorectal cancer varies in different high-risk conditions. Ann Med 1998;30:75-80.

13 Kim H, Jen J, Vogelstein B, et al. Clinical and pathological characteristics of sporadic colorectal carcinomas with DNA replication errors in microsatellite sequences. $A m \mathcal{F}$ Pathol 1994;145:148-56.

14 Risio M, Reato G, di Celle PF, et al. Microsatellite instability is associated with the histological features of the tumor in nonfamilial colorectal cancer. Cancer Res 1996;56:5470in 4 .

15 Thibodeau SN, French AJ, Cunningham JM, et al. Microsatellite instability in colorectal cancer: different mutato phenotypes and the principal involvement of hMLH1 Cancer Res 1998;58:1713-18.

16 Veigl ML, Kasturi L, Olechnowicz J, et al. Biallelic inactivation of hMLH1 by epigenetic gene silencing, a novel mechanism causing human MSI cancers. Proc Natl Acad Sci USA 1998;95:8698-702

17 Herman JG, Umar A, Polyak K, et al. Incidence and functional consequences of hMLH1 promoter hypermethylation in colorectal carcinoma. Proc Natl Acad Sci USA 1998;95:6870-5.

18 Cunningham JM, Christensen ER, Tester DJ, et al. Hypermethylation of the hMLH1 promoter in colon cancer with microsatellite instability. Cancer Res 1998;58: 3455-60.

19 Lothe RA, Peltomaki P, Meling GI, et al. Genomic instability in colorectal cancer: relationship to clinicopathological ity in colorectal cancer: relationship to clinicopathological
variables and family history. Cancer Res 1993;53:5849-52.

20 Bubb VJ, Curtis LJ, Cunningham C, et al. Microsatellite instability and the role of hMSH2 in sporadic colorectal instability and the role of hMSH

21 Brown SR, Finan PJ, Hall NR, et al. Incidence of DNA replication errors in patients with multiple primary cancers Dis Colon Rectum 1998;41:765-9.

22 Cawkwell L, Gray S, Murgatroyd H, et al. Choice of management strategy for colorectal cancer based on a diagnostic immunohistochemical test for defective mismatch repair. Gut 1999;45:409-15.

23 Halling KC, French AJ, McDonnell SK, et al. Microsatellite instability and $8 \mathrm{p}$ allelic imbalance in stage B2 and C colorectal cancers. F Natl Cancer Inst 1999;91:1295-303.

24 Messerini L, Ciantelli M, Baglioni S, et al. Prognostic significance of microsatellite instability in sporadic mucinous colorectal cancers. Hum Pathol 1999;30:629-34.

25 Salahshor S, Kressner U, Fischer H, et al. Microsatellite instability in sporadic colorectal cancer is not an independinstability in sporadic colorectal cancer is not an in

26 Feeley KM, Fullard JF, Heneghan MA, et al. Microsatellite instability in sporadic colorectal carcinoma is not an instability in sporadic colorectal carcinoma

27 Curran B, Lenehan K, Mulcahy H, et al. Replication error phenotype, clinicopathological variables, and patient outcome in Dukes' B stage II (T3,N0,M0) colorectal cancer Gut 2000;46:200-4

28 Jernvall P, Makinen MJ, Karttunen TJ, et al. Microsatellite instability: impact on cancer progression in proximal and distal colorectal cancers. Eur f Cancer 1999;35:197-201.

29 Masubuchi S, Konishi F, Togashi K, et al. The significance of microsatellite instability in predicting the development of metachronous multiple colorectal carcinomas in patients with nonfamilial colorectal carcinoma. Cancer 1999;85: 1917-24.

30 Sengupta SB, Yiu CY, Boulos PB, et al. Genetic instability in patients with metachronous colorectal cancers. Br f Surg 1997;84:996-1000.

31 Carethers JM, Chauhan DP, Fink D, et al. Mismatch repair proficiency and in vitro response to 5-fluorouracil. Gastroenterology 1999;117:123-31.

32 Colella G, Marchini S, Di M, et al. Mismatch repair deficiency is associated with resistance to DNA minor groove alkylating agents. Br f Cancer 1999;80:338-43.

33 Fink D, Aebi S, Howell SB. The role of DNA mismatch repair in drug resistance. Clin Cancer Res 1998;4:1-6.

34 Iino H, Jass JR, Simms LA, et al. DNA microsatellite instability in hyperplastic polyps, serrated adenomas, and mixed polyps: a mild mutator pathway for colorectal cancer? $\mathcal{f}$ Clin Pathol 1999;52:5-9.

35 Hermanek P, Sobin LH. TNM. American foint Committee on Cancer, manual for staging of cancer, 5 th edn. Philadelphia: Lippincott-Raven, 1998.

36 Offit K. Clinical cancer genetics. New York: John Wiley and Sons, 1998

37 Jass JR, Sobin LH. Histological typing of intestinal tumours. WHO international classification of tumours, 2 nd edn. Berlin: Springer-Verlag, 1989.

38 Jass JR, Ajioka Y, Allen JP, et al. Assessment of invasive growth pattern and lymphocytic infiltration in colorectal cancer. Histopathology 1996;28:543-8.

39 Boland CR, Thibodeau SN, Hamilton SR, et al. A National Cancer Institute Workshop on Microsatellite Instability for cancer detection and familial predisposition: development of international criteria for the determination of microsatellite instability in colorectal cancer. Cancer Res 1998;58: 5248-57.

40 Ward RL, Hawkins NJ, O'Grady R, et al. Restriction endonuclease-mediated selective polymerase chain reaction: a novel assay for the detection of $\mathrm{K}$-ras mutations in clinical samples. Am F Pathol 1998;153:373-9.

41 Ward RL, Todd AV, Santiago F, et al. Activation of the K-ras oncogene in colorectal neoplasms is associated with decreased apoptosis. Cancer 1997;79:1106-13.

42 Lamberti C, Kruse R, Ruelfs C, et al. Microsatellite instability-a useful diagnostic tool to select patients at high risk for hereditary non-polyposis colorectal cancer: a study in different groups of patients with colorectal cancer. Gut 1999;44:839-43.

43 Liu B, Farrington SM, Petersen GM, et al. Genetic instability occurs in the majority of young patients with colorectal cancer. Nat Med 1995;1:348-52.

44 Lukish JR, Muro K, DeNobile J, et al. Prognostic significance of DNA replication errors in young patients with colorectal cancer. Ann Surg 1998;227:51-6.

45 Gryfe R, Kim H, Hsieh ET, et al. Tumor microsatellite instability and clinical outcome in young patients with instability and clinical outcome in young patient

46 Fujiwara T, Stolker JM, Watanabe T, et al. Accumulated clonal genetic alterations in familial and sporadic colorectal carcinomas with widespread instability in microsatellite sequences. Am f Pathol 1998;153:1063-78.

47 Dietmaier W, Wallinger S, Bocker T, et al. Diagnostic microsatellite instability: definition and correlation with mismatch repair protein expression. Cancer Res 1997;57:474956.

48 Wright $\mathrm{CM}$, Dent OF, Barker $\mathrm{M}$, et al. Prognostic significance of extensive microsatellite instability in spo-
radic clinicopathological stage $\mathrm{C}$ colorectal cancer. $\mathrm{Br} \mathcal{F}$ radic clinicopathological
Surg 2000;87:1197-202.

49 Jass JR, Do KA, Simms LA, et al. Morphology of sporadic colorectal cancer with DNA replication errors. Gut 1998;42:673-9.

50 Thibodeau SN, French AJ, Roche PC, et al. Altered expression of hMSH 2 and hMLH1 in tumors with microsatellite instability and genetic alterations in mismatch repair genes. Cancer Res 1996;56:4836-40.

51 Curia MC, Palmirotta R, Aceto G, et al. Unbalanced germline expression of $\mathrm{hMLH} 1$ and $\mathrm{hMSH} 2$ alleles in hereditary nonpolyposis colorectal cancer. Cancer Res 1999;59:3570-5.

52 Akiyama Y, Sato H, Yamada T, et al. Germ-line mutation of the hMSH6/GTBP gene in an atypical hereditary nonpolyposis colorectal cancer kindred. Cancer Res 1997;57:39203.

$53 \mathrm{Ku} \mathrm{JL}$, Yoon KA, Kim DY, et al. Mutations in hMSH6 alone are not sufficient to cause the microsatellite instability in 